\title{
ANALYTICAL SPECTRA OF CMB ANISOTROPIES AND POLARIZATION
}

\author{
YANG ZHANG* and ZHENG CAI \\ Key Laboratory for Researches in Galaxies and Cosmology, Center for Astrophysics \\ University of Science and Technology of China \\ Hefei, Anhui, 230026, China \\ *yzh@ustc.edu.cn
}

\begin{abstract}
We briefly present the results of our analytical studies on CMB anisotropies and polarizations generated by scalar metric perturbations in synchronous gauge, parallel to the previous work with RGW as a generating source. The analytical the spectra $C_{l}^{X X}$ are explicitly given and the influences of various cosmological processes are examined.
\end{abstract}

Keywords: $98.70 \mathrm{Vc}, 98.80 .-\mathrm{k}, 98.80 . \mathrm{Jk}$

The temperature anisotropies and polarization of the cosmic microwave background radiation $(\mathrm{CMB})$ are the indispensable cosmological probes. ${ }^{1,2}$ There have been codes of numerical computation for the spectra $C_{l}^{X X}$, such as $\mathrm{CMBFAST}^{3}$ and CAMB. ${ }^{4}$ Analytical studies are still useful for understanding how various underlying physical effects give rise to the observed patterns. In particular, the analytical $C_{l}^{X X}$ are helpful to separate the contribution by the relic gravitational waves $(\mathrm{RGW})^{5}$ from that by the density perturbation. ${ }^{6}$ In the past we systematically derived the analytic $C_{l}^{X X}$ induced by $\mathrm{RGW}^{7-9}$ and their modifications due to reionization. ${ }^{10,11}$ I briefly present the analytic $C_{l}^{X X}$ induced by the density perturbations in synchronous gauge from our recent studies.

The Boltzmann equation of photons consists of two coupled differential equations for the temperature anisotripies $\alpha_{k}$ and the polarization $\beta_{k}$ for each mode $k,{ }^{12}$

$$
\begin{gathered}
\left(\frac{\partial}{\partial \tau}+q(\tau)+i k \mu\right) \alpha_{k}=\frac{1}{2}\left(\frac{d H}{d \tau}-\mu^{2} \frac{d H_{l}}{d \tau}\right)+q(\tau)\left(\mathcal{I}_{1}(\tau)+i \mu v_{b}-\frac{1}{2} P_{2}(\mu) \mathcal{I}_{2}(\tau)\right), \\
\left(\frac{\partial}{\partial \tau}+q(\tau)+i k \mu\right) \beta_{k}=\frac{1}{2} q(\tau)\left(1-P_{2}(\mu)\right) \mathcal{I}_{2}(\tau),
\end{gathered}
$$

where $\mu=\cos \theta$ with $\theta$ being the angle between the direction of photon propagation and the wave vector $\mathbf{k}, q(\tau)$ is the differential optical depth for recombination, $H(\tau)$ and $H_{l}(\tau)$ are the two modes of scalar metric perturbations and $\frac{1}{2}\left(\frac{d H}{d \tau}-\mu^{2} \frac{d H_{l}}{d \tau}\right)$ is the Sachs-Wolfe term. $P_{2}(\mu)$ is the second order Legendre function, and $\mathcal{I}_{1}(\tau) \equiv$ $\frac{1}{2} \int_{-1}^{1} d \mu \alpha_{k}(\tau, \mu), \mathcal{I}_{2}(\tau) \equiv \frac{1}{2} \int_{-1}^{1} d \mu\left[\left(1-P_{2}(\mu)\right) \beta_{k}(\tau, \mu)-P_{2}(\mu) \alpha_{k}(\tau, \mu)\right]$ play a role 
of sources. $q\left(\mathcal{I}_{1}+i \mu v_{b}-\frac{1}{2} P_{2} \mathcal{I}_{2}\right)$ with $v_{b}$ being the baryon (electron) velocity in Eq. (1) is a new collision term for $\alpha_{k}$, which is absent in the case of RGW.

Equations (1) and (2) are analytically solved by time-integration in the long wave-length limit, resulting in explicit expressions of $\alpha_{k}$ and $\beta_{k}$. Several techniques have been employed in achieving this. The tightly-coupling approximation for the photons during the recombination process has been used. A two-piece half-gaussian function is used for the visibility function during the recombination. The long wavelength approximation for the modes $H$ and $H_{l}$ during the radiation dominant era is used, and the residual gauge modes have been removed from the solution of $H$ and $H_{l}$ in the synchronous gauge. A proper connection has been made for $H$ and $H_{l}$, respectively, at the radiation-matter equality.

The analytical multipole moment of temperature anisotropies

$$
\begin{aligned}
a_{l}^{T}(k) \simeq & \alpha_{0}\left(\tau_{d}\right) D_{T}(k) j_{l}\left(k\left(\tau_{0}-\tau_{d}\right)\right)+\alpha_{1}\left(\tau_{d}\right) D_{T}(k) \frac{d}{d \zeta} j_{l}\left(k\left(\tau_{0}-\tau_{d}\right)\right. \\
& +\left(H\left(\tau_{0}\right)-H\left(\tau_{d}\right)\right) j_{l}\left(k \tau_{0}\right) \\
& -\left.\frac{15}{14} \ln \frac{10}{3} \Delta \tau_{d} M\left(\tau_{d}\right) D_{E}(k)\left(1+\frac{d^{2}}{d \zeta^{2}}\right) j_{l}(\zeta)\right|_{\zeta=k\left(\tau_{0}-\tau_{d}\right)},
\end{aligned}
$$

where $\tau_{0}$ is the present time, $\tau_{d}$ is the recombination time, and $\Delta \tau_{d}$ is the recombination width, and the damping factor $D_{T}(k)=\frac{1}{2}\left(e^{-c_{T}\left(k \Delta \tau_{1}\right)^{b} T}+e^{-c_{T}\left(k \Delta \tau_{2}\right)^{b} T}\right)$ with $c_{T} \sim 0.65$ and $b_{T} \sim 0.6, D_{E}(k)=0.2\left(e^{-c_{E}\left(k \Delta \tau_{1}\right)^{b} E}+e^{-c_{E}\left(k \Delta \tau_{2}\right)^{b} E}\right)$ with $c_{E} \sim 0.27$ and $b_{E} \sim 2.0$, and $M(\tau) \equiv-\frac{2}{5} \dot{\alpha}_{0}(\tau)+\frac{2}{5} \dot{H}(\tau)$. In Eq. (3), the monopole $\alpha_{0}$ term is dominant, the dipole $\alpha_{1}$ term is secondary, the ISW term is smaller than the $\alpha_{1}$ term, and the last term is the quadrupole part, which is smaller than the ISW term. We have derived the explicit expressions of $\alpha_{0}(\tau), \alpha_{1}(\tau), H(\tau), H_{l}(\tau)$ which are not written down here for a limited length of paper. The analytical multipole moment of polarization

$$
a_{l}^{E}(k) \simeq \frac{15}{14} \ln \frac{10}{3}\left(\frac{(l+2) !}{(l-2) !}\right)^{1 / 2} \frac{\Delta \tau_{d}}{k^{2}\left(\tau_{0}-\tau_{d}\right)^{2}} M\left(\tau_{d}\right) D_{E}(k) j_{l}\left(k\left(\tau_{0}-\tau_{d}\right)\right),
$$

which is proportional to $M\left(\tau_{d}\right) \Delta \tau_{d}$. Since $\Delta \tau_{d}$ is small, the amplitude of $a_{l}^{E}$ will be consequently small, in comparison with $a_{l}^{T}$. Notice that the quadrupole part of $a_{l}^{T}$ contains the factor $M\left(\tau_{d}\right) \Delta \tau_{d}$, similar to the polarization $a_{l}^{E}$ in Eq. (4).

These new results significantly extend the earlier preliminary works. The analytic expression $a_{l}^{T}$ extends those in Refs.13 in Newtonian gauge, and fulfils what was not completed in Ref.12, and, to a great extent, improves what was given in Ref.14. The analytic expressions of polarization $a_{l}^{E}$ and the related spectra $C_{l}^{E E}$, and $C_{l}^{T E}$ are what have not been addressed in Refs.13, 14. The spectra are calculated as the integrations over the wavenumber $k, C_{l}^{T T}=\int\left|a_{l}^{T}\right|^{2} k d k, C_{l}^{T E}=\int a_{l}^{T} a_{l}^{E} k d k$, $C_{l}^{E E}=\int\left|a_{l}^{E}\right|^{2} k d k$. Many features are revealed by these analytic expressions, here we only mention a few of them.

Figure 1 shows the analytical spectra $l(l+1) C_{l}^{X X}$. The first two peaks of $C_{l}^{T T}$ occur at $l \sim 200$ and $l \sim 500$, while those of $C_{l}^{E E}$ occur at $l \sim 100$ and $l \sim 400$. This 

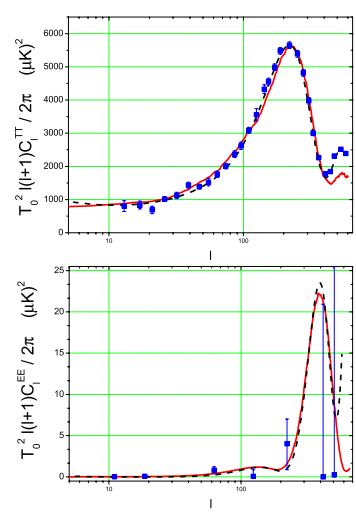

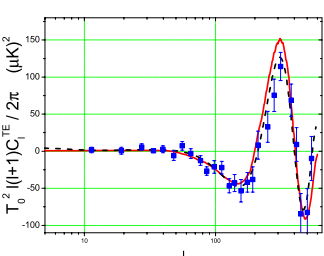

squares: WMAP5

dash : CAMB

solid: analytical

Fig. 1. The analytic spectra (solid line) compared with the numerical ${ }^{4}$ and the observed ${ }^{2}$ for the scalar index $n_{s}=0.96$, the running index $\alpha_{s}=0$, and the baryon fraction $\Omega_{b}=0.045$.

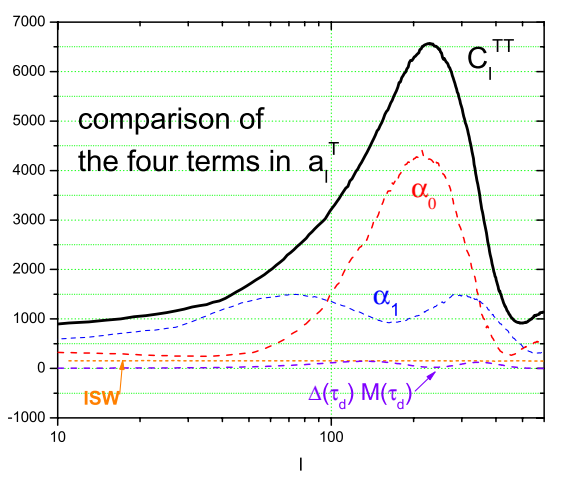

Fig. 2. The four terms, $\alpha_{0}, \alpha_{1}$, the ISW , and $M\left(\tau_{d}\right) \Delta \tau_{d}$ in Eq. (3) are schematically shown for comparison.

alternating occurrence of the peak locations has been anticipated from our analytic results. The overall profiles of the analytical spectra agree well with the observed result from WMAP $5^{2}$ and numerical result from $\mathrm{CAMB}^{4}$ on large angular scales with $l \lesssim 500$. On smaller angular scales, the analytical results deviate considerably from the numerical ones. This has been expected since our calculation is based upon the long wavelength approximation.

Figure 2 demonstrates the contributions by each term to $a_{l}^{T}$ in Eq. (3). The contribution by ISW is at most $\sim 10 \%$ that of the $\alpha_{1}$ term. The contribution by the last term in Eq. (3) is even smaller than the ISW term since $\Delta \tau_{d} \sim 0.003$ is small, with two low bumps at $l \sim 130$ and at $l \sim 350$. Thus, the overall profile of $a_{l}^{T}$ is largely determined by $\alpha_{0}\left(\tau_{d}\right)$ and $\alpha_{1}\left(\tau_{d}\right)$. 


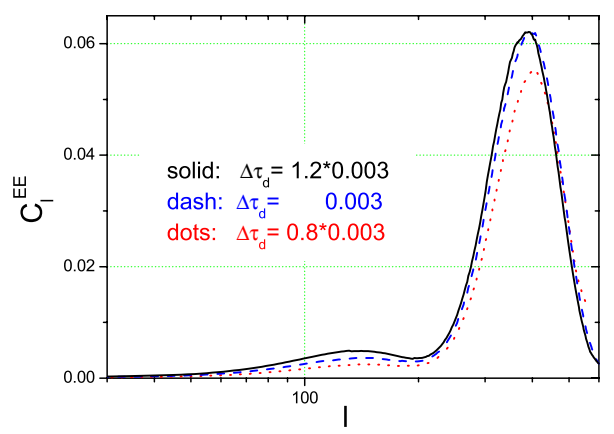

Fig. 3. A greater $\Delta \tau_{d}$ yields a higher amplitude of $C_{l}^{E E}$, and brings more small-scale damping.

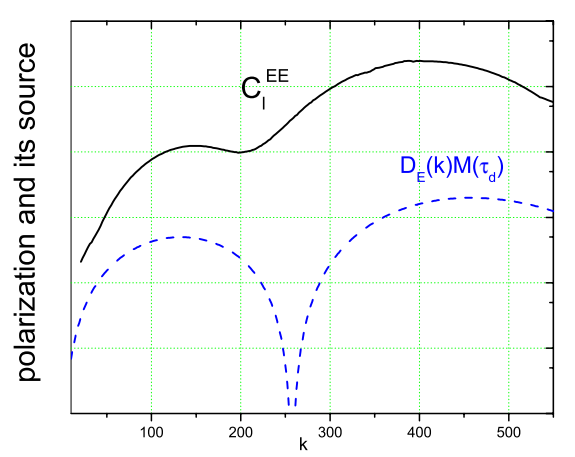

Fig. 4. The two bump locations of $C_{l}^{E E}$ is determined by $M\left(\tau_{d}\right)$.

Figure 3 tells that a longer recombination process (a greater $\Delta \tau_{d}$ ) yields a higher amplitude of polarization, since $a_{l}^{E} \propto \Delta \tau_{d}$ by Eq. (4). It also shows that a longer recombination process brings more damping of $C_{l}^{E E}$ on small scales. This is because $a_{l}^{E}$ in Eq. (4) contains the damping factor $D_{E}(k) \propto e^{-c_{E}\left(k \Delta \tau_{d}\right)^{2}}$. Similarly, this feature also is shared by $C_{l}^{T T}$. These properties may help to constrain the recombination width $\Delta \tau_{d}$ by the observational data.

In Figure 4, we sketch the profile of $l(l+1) C_{l}^{E E}$ as a function of $l$, which has two bumps, one at $l \sim 100$, and another at $l \sim 400$. In order to interpret the origin of these two bumps, we also sketch the main factor $D_{E}(k) M\left(\tau_{d}\right) /\left[k\left(\tau_{0}-\tau_{d}\right)\right]^{2}$ of $a_{l}^{E}$ in Eq. (4) as a function of $k$. It is $D_{E}(k) M\left(\tau_{d}\right)$ with two bumps around $k \sim 100$ and $k \sim 400$ that gives rise to the two bumps of $C_{l}^{E E}$. 


\section{Acknowledgments}

Y. Zhang would like to thank the organizers of the $3^{r d}$ Galileo - Xu Guangqi meeting.

The work is supported by the CNSF No.10773009, SRFDP, and CAS.

\section{References}

1. E. Komatsu, et al, Astrophys. J. Suppl. 180 (2009) 330.

2. M. R. Nolta, et al., Astrophys. J. Suppl. Ser. 180 (2009) 296.

3. U. Seljak and M. Zaldarriaga, Astrophys. J. 469 (1996) 437.

M. Zaldarriaga, U. Seljak, E. Bertschinger, Astrophys. J. 494 (1998) 491.

M. Zaldarriaga and U. Seljak, Astrophys. J. 129 (2000) 431.

The cmbfast Online Tool can be available at http://lambda.gsfc.nasa.gov/toolbox/tb_cmbfast_form.cfm

4. A. Lewis, A. Challinor and A. Lasenby, Astrophys. J. 538 (2000) 473. http://lambda.gsfc.nasa.gov/toolbox/tb_camb_form.cfm

5. M. L. Tong and Y. Zhang, Phys. Rev. D 80 (2009) 084022.

H. X. Miao and Y. Zhang, Phys. Rev. D 75 (2007) 104009.

S. Wang, Y. Zhang, T. Y. Xia and H. X. Miao, Phys. Rev. D 77 (2008) 104016.

6. M. Basko and A. Polnarev, Mon. Not. R. Astron. Soc. 191 (1980) 207.

M. Zaldarriaga and U. Seljak, Phys. Rev. D 55 (1997) 1830.

7. W. Zhao and Y. Zhang, Phys. Rev. D 74 (2006) 083006.

8. T. Y. Xia and Y. Zhang, Phys. Rev. D 78 (2008) 123005.

9. Y. Zhang, et al., Int.JMPD Vol 17 (2008) 1105.

10. T. Y. Xia and Y. Zhang, Phys. Rev. D 80 (2009) 083002.

11. Y. Zhang, et al., Int.JMPD Vol 20 (2011) 2099.

12. D. Baskaran, L. P. Grishchuk, A. G. Polnarev, Phys. Rev. D 74 (2006) 083008.

A. Polnarev, N. Miller and B. Keating, Mon. Not. R. Astron. Soc. 386 (2008) 1053;

B. Keating, A. Polnarev, N. Miller, D. Baskaran, Int.JMPA 21 (2006) 2459.

13. W. Hu and N.Sugiyama, Astrophys. J. 444 (1995) 489.

14. S. Weinberg, Phys. Rev. D 64 (2001) 123511; Phys. Rev. D 64 (2001) 123512. 